\title{
Factors associated with type 2 diabetes mellitus treatment choice across four
}

\section{European countries}

Authors: Edith M. Heintjes ${ }^{1}$, Jetty A. Overbeek ${ }^{1}$, Gillian C. Hall ${ }^{2}$, Daniel Prieto-Alhambra ${ }^{3,4}$, Francesco Lapi $^{5}$, Niklas Hammar ${ }^{6,7}$, Irene D. Bezemer ${ }^{1}$

${ }^{1}$ PHARMO Institute for Drug Outcomes Research, Utrecht, the Netherlands; ${ }^{2}$ Grimsdyke House, London, United Kingdom; ${ }^{3}$ GREMPAL Research Group and CIBERFes, Idiap Jordi Gol Primary Care Research Institute, Universitat Autonoma de Barcelona and Instituto de Salud Carlos III, Barcelona, Spain; ${ }^{4}$ Centre for Statistics in Medicine, NDORMS, University of Oxford, Oxford, UK; ${ }^{5}$ Health Search, Italian College of General Practitioners and Primary Care, Florence, Italy; ${ }^{6}$ AstraZeneca R\&D, MöIndal, Sweden, ${ }^{7}$ Institute of Environmental Medicine, Karolinska Institutet, Stockholm, Sweden

Corresponding Author: Mrs Edith M Heintjes, PhD, PHARMO Institute for Drug Outcomes Research

\section{$\underline{\text { Abstract }}$}

Purpose: To identify factors associated with the choice of type 2 diabetes mellitus (T2DM) therapy at the time of intensification of antidiabetic treatment across four European countries.

Methods: For T2DM patients from the Netherlands (NL), Italy (IT), Spain (ES) (2007-2011) and United Kingdom (UK) (2008-2012) antidiabetic drug prescription/dispensing records and patient characteristics were obtained from electronic health-care records. Oral monotherapy was defined as $1^{\text {st }}$ line, oral dual therapy as 2 nd line, $>2$ oral treatments or oral combined with an injectable as 3 rd line and injectables only as 4 th line treatment. Treatment intensification was defined as starting a higher line of treatment. Comedication, comorbidities, clinical parameters and other factors associated with treatment choice were identified using multivariable relative risk estimation by Poisson regression with robust error variance.

Findings: In the study period 485,120 patients, i.e. $79 \%$ of the treated T2DM population underwent treatment intensification. Changes in treatment choice were clearly visible over the study period, such as decline of thiazolidinedione (TZD) use (NL, ES, UK), and increase of dipeptidyl peptidase-4 inhibitors (DPP4i) use (NL, ES, UK) and glucagon-like peptide-1 receptor agonist (GLP-1ra) use (UK). For 1st line treatment advanced age and renal comorbidity were associated with SU (all countries), whereas high BMI was inversely associated with SU in UK and ES. For 2 nd line advanced age was associated with metformin + SU (all countries) and renal comorbidity with SU + dipeptidyl peptidase4 inhibitors (DPP4i) in UK and NL. High BMI was associated with metformin + thiazolidinedione (TZD) in UK and ES, and with metformin + DPP4i (UK).

For 3rd line advanced age and renal comorbidity were associated with SU + insulin (NL, ES, UK). $\mathrm{HbA1c} \geq 8.5 \%$ was positively and high BMI inversely associated with any $3 \mathrm{rd}$ line combination containing insulin. For 4 th line treatment women were more likely to receive GLP-1ra than men in UK and ES.

Implications: The results suggest that the main factors driving treatment choice at any stage of intensification were age, $\mathrm{HbA1c}, \mathrm{BMI}$, renal and cardiac morbidity, and prior treatment history. These drivers were consistent with guidelines and contra-indications for specific medications. 
Differences between countries were generally consistent with, but not solely attributable to differences in local guidelines and reimbursement policies.

Keywords: diabetes mellitus, type 2; glucose lowering drugs; treatment

\section{Conflicts of Interest statement}

Drs. Overbeek, Dr. Heintjes, Dr. Bezemer and Dr. Herings are employees of the PHARMO Institute for Drug Outcomes Research. This independent research institute performs financially supported studies for government and related healthcare authorities and several pharmaceutical companies. Dr. Prieto-Alhambra's research group and/or department have received unrestricted research grants from Bioberica, AMGEN, Servier Laboratoires, and UCB Biopharma. Dr. Hall has been a member of the Advisory Group of the THIN database and has received funding for research and consultancy from a number of pharmaceutical companies and from charities. Dr. Lapi provided consultancies in protocol preparation and data analysis for epidemiologic studies for AMGEN, Sanofi, and Eli-Lilly. Dr. Hammar is an employee of AstraZeneca. The authors have indicated that they have no other conflicts of interest regarding the content of this article. 


\section{INTRODUCTION}

Type 2 diabetes mellitus (T2DM) is a progressive disease of epidemic proportions. The estimated prevalence of diabetes in Europe is expected to rise from 9.1\% (59.8 million patients) in 2015 to 10.7\% (71.1 million patients) in 2040. In Western Europe the estimated prevalence in 2015 ranges from $<5 \%$ to $>9 \% 1$. Cardiovascular and other complications may be prevented by close monitoring of blood glucose levels and intensification of treatment if glycemic targets are not met.

The increasing complexity and recognized importance of glucose lowering treatment has led to development of guidelines to help physicians make treatment decisions. Despite the international ADA/EASD consensus statement for the T2DM treatment algorithm of 20062 and subsequent position statements3,4, European countries have developed local guidelines as well5-8, each weighing cost considerations and the evidence of effectiveness and risk differently, resulting in slightly different recommendations. The general consensus at the time of this study was that intensification of treatment based on failure to reach glycemic targets should take place along the following lines: start with oral monotherapy, preferably metformin. If that is no longer effective, subsequent treatment steps are adding a second oral drug, and subsequently adding a third oral drug, basal insulin or a glucagon-like peptide-1 receptor agonist (GLP-1ra). Most guidelines allow skipping steps in the above mentioned algorithm when HbA1c levels are far from the target of $7 \%$ (53 mmol/mol). Insulin or GLP-1ra agonist monotherapy may be considered if patients do not tolerate or seem to benefit from a combination with oral drugs, but it is not recommended in the guidelines, as oral drugs may help to keep insulin doses low.

There is variation in local guidelines, which could result in different treatment choices across countries. Published treatment pattern studies 9,10 mainly describe cross-sectional distributions of prescribed diabetes treatments rather than factors associated with treatment choice. In a previous study11 we described individual switching patterns in the period 2007-2012 in 5 European countries.

\section{METHODS}

\section{Setting}

Data for this observational cohort study were obtained from population-based databases from four European countries containing electronic health records from routine primary care: the PHARMO Database Network12 in the Netherlands, the Health Search longitudinal patient Database (HSD) in Italy , the Sistema d' Informació per al Desenvolupament de la Investigació en Atenció Primària13,14 (SIDIAP) in Catalonia, Spain and The Health Improvement Network (THIN) in the United Kingdom (UK)15. Additional information regarding the different data sources is described elsewhere11.

\section{Study population}

The source population included all adult patients with T2DM receiving glucose lowering treatment during the most recent five-year period that was available in each database at the time of analysis: 2007-2011 for the Netherlands (NL), Spain (ES), and Italy (IT), and 2008-2012 for the UK. T2DM patients were defined as having a diagnosis code for T2DM or at least two prescriptions for noninsulin blood glucose lowering drugs within a six-month period at any time in the available medication records. Patients with a recorded diagnosis code for type 1 diabetes mellitus or for gestational diabetes were excluded. 
Treatment patterns were determined as described in a prior publication11. Drug prescription records were assigned to the oral drug classes metformin, sulfonylureas (SU), thiazolidinediones (TZD), dipeptidyl peptidase 4 inhibitors (DPP4i), and 'other' (e.g. alpha-glucosidase inhibitors and meglitinides), or the injectable classes of GLP-1ra or insulin. Use of a drug class was assumed uninterrupted if prescriptions were maximally 9 months apart. Overlap in episodes of use of different classes constituted combined use. If a new drug class was started within 9 months of a prior class and there were no repeat prescriptions of the prior class, the prior class stopped at the time the new class started. If prescriptions of any class were absent for 9 months, treatment was considered to have stopped one month after the last prescription preceding those 9 months.

First line treatment was defined as oral monotherapy, 2nd line treatment as dual oral therapy, 3rd line treatment as triple oral therapy or oral/insulin or oral/GLP-1ra combination therapy, and 4th line therapy as insulin or GLP-1ra monotherapy or combination therapy without oral drugs. Treatment intensification was defined as the prescription/drug dispensing of a new line of treatment preceded only by lower lines or no therapy. Patients with treatment intensification within the study period were included in the analyses provided they had $\geq 12$ months of continuous enrolment immediately preceding the intensification date in order to determine patient characteristics. Patients could be included in analyses of multiple intensifications.

Data management

All database custodians were asked to prepare input files following a common data model. All data were analysed at the site of the database custodian using a common SAS analysis tool creating treatment patterns and automated SAS programs for evaluation of factors associated with treatment choice, developed at the site of the principle investigator (PHARMO). The principal investigator combined the aggregated results from all countries. All sites used SAS ${ }^{\circledR}$ version 9.2 or 9.4 (SAS Institute Inc., Cary, NC, USA) in a Windows environment.

Considered treatments

Per line of treatment specific intensifications were reported if in all contributing countries they represented at least 100 patients or $5 \%$ of the study population starting the line of treatment. The following treatments were considered for analysis: for 1st line (by definition new users): metformin, SU, TZD, DPP4i, other OAD, GLP-1ra and insulin; for 2nd line: combined metformin \& SU, any oral combination treatment including TZD and any oral combination treatment including DPP4i; for $3^{\text {rd }}$ line: frequently used combinations of at least three oral drugs, oral + insulin and oral + GLP-1ra; and for 4th line: insulin only, GLP-1ra only, and the combination of insulin and GLP-1ra.

\section{Patient characteristics}

For all patients the following general characteristics were determined at each intensification date: gender, age and year of intensification. Furthermore, prior antidiabetic treatment, comedication (use of lipid lowering drugs, antihypertensive drugs, cardiac drugs, anticoagulants and COPD/asthma drugs), comorbidities (microvascular (diabetic foot/peripheral vascular disease (PVD)/neuropathy, retinopathy, and renal complications), macrovascular (coronary artery disease and cerebrovascular disease), and other comorbidities (ulcers at other locations, other cardiovascular disease, chronic respiratory disease, cancer, urinary tract infections, and mental disease) and clinical parameters (BMI, cardiovascular risk assessment, systolic blood pressure (SBP), diastolic blood pressure (DBP), $\mathrm{HbA} 1 \mathrm{c}$ and chronic kidney disease (CKD)) were determined. Prior antidiabetic treatment was based on the episodes of mono- and or combination treatment directly leading up to intensification and comedication was based on the prescription records during the 6 months prior to the intensification 
date, but after the previous change. All comorbidities were based on GP recorded diagnoses, primary care laboratory results and/or hospitalisations determined in the entire available history prior to the intensification date. Clinical parameters were based on GP recorded information; tobacco smoking, BMI and cardiovascular risk assessment were assessed in the entire available history prior to intensification date, SBP, DBP, HbA1c, lipids and CKD were assessed during the prior antidiabetic treatment in the 6 months prior to the intensification date.

\section{Statistical analysis}

The proportions of patients per country, per line and per treatment class were reported descriptively. General characteristics, prior antidiabetic treatment, comedication, comorbidities and clinical parameters at intensification are reported descriptively, presenting proportions for categorical variables, and mean with standard deviation (SD) or median with interquartile range (IQR) for continuous variables.

Relative Risk Estimation by Poisson Regression with Robust Error Variance was used to obtain relative risks (RR) and $95 \%$ confidence intervals 16 of patient characteristics (independent variables) associated with choice of treatment within each line of treatment (dependent variable). Dummy variables were created to allow comparison of each therapy to all alternatives within a line of treatment. Unadjusted and adjusted RR were presented. For the multivariate model a backward selection method was used, forcing age into the model, considering all variables with a univariate association at $p<0.20$ and retaining them at $p<0.10$ in the multivariate model. Missing values were included in the models as a separate category.

\section{RESULTS}

Patients and treatment characteristics Overall, 617,346 patients received T2DM treatment during the study period, 485,120 (79\%) of whom were included in the current study cohort as they initiated or intensified treatment within the study period. Spain contributed $61 \%$, UK $22 \%$, Italy $9 \%$ and the Netherlands $7 \%$ to the study cohort. Combining all countries, $47 \%$ initiated 1 st line-treatment, $28 \%$ intensified to 2 nd line-treatment, $19 \%$ intensified to 3 rd line-treatment and $7 \%$ to 4 th linetreatment. A relatively large proportion of Italian patients used 4 th line treatment $(11 \%)$, whilst this proportion was small in the UK (3\%) (Table 1).

Figure 1 shows the distribution of treatments observed per intensification. Across all countries, metformin was the most common 1st line treatment, ranging from $79 \%$ (IT) to $93 \%$ (UK), followed by SU, ranging from $7 \%$ (UK) to $14 \%$ (IT). At initiation of 2 nd line, the majority of patients were treated with a combination of metformin and SU, ranging from $62 \%$ (IT) to $85 \%$ (NL). For 3rd line treatment, combinations of metformin and SU with a third drug were most common, but the third drug differed across countries: insulin was most common in the Netherlands (34\%), DPP4i in the UK and Spain (33\% and $28 \%$, respectively) and TZD in Italy (21\%). The proportion of 3rd line treatments with insulin was $22 \%$ in the UK, $46 \%$ in Italy, $49 \%$ in Spain and $71 \%$ in the Netherlands. GLP-1ra agonists were part of $\sim 3 \%$ of the 3 rd line treatments in Italy, Spain and the Netherlands, but $12 \%$ in the UK. Insulin was the most common 4th line therapy for all countries, ranging from $94 \%$ (UK) to $99 \%$ (NL); the combination of GLP-1ra agonists with insulin at initiation of 4 th line treatment was rare $(<0.1 \%)$.

Table 1 shows the patient characteristics at intensification. By definition, none of the patients initiating first line had prior antidiabetic treatment. Proportions of patients without treatment registered in GP records in the 9 months prior to intensification were also considerable among other lines of treatment: $17 \%$ starting 2 nd line, $8 \%$ starting 3rd line and $37 \%$ starting 4 th line. The most frequent antidiabetic drug treatments prior to intensification were metformin (57\%) or SU (23\%) for 
2nd line, metformin and SU (49\%) or oral monotherapy (21\%) for 3rd line, and metformin + SU (32\%) or oral monotherapy $(20 \%)$ for 4 th line.

Frequencies of cardiovascular co-medication and micro- or macrovascular comorbidities increased with each intensification up to $3 r d$ line. At the 4 th line more renal complications, macrovascular comorbidities, cancer, cardiac and respiratory drug use were observed, but less lipid lowering and antihypertensive drug use compared to $3 \mathrm{rd}$ line. BMI and $\mathrm{HbA1c}$ were more often recorded before initiating higher lines of treatment. Furthermore, BMI seemed to decrease with more intense treatment and $\mathrm{HbA1c}$ increased from 1st to 3rd line of treatment. In all treatment lines information on cardiovascular risk assessment, blood pressure and $\mathrm{HbA1c}$ was sparse.

\section{Factors associated with treatment choice}

The adjusted RR for factors associated with treatment choice are presented in figures 2-5. Different variables were retained in the models of different treatments for different countries $(p<0.1)$. For the sake of clarity, some variables with relative risks between 0.9 and 1.1 across all treatments across all represented countries were removed from the graphs, the complete lists of associated variables with RR can be found in the supplement online supplemental tables.

\section{$\underline{1 s t}$ line}

Figure 2 shows the factors associated with the choice for $\mathrm{SU}$, the second most common 1st line therapy, versus any alternative, which was usually metformin. Factors most strongly associated were age ( $\geq 75$ years 2.0 to 3.7 times more likely), BMI ( $\geq 30 \mathrm{~kg} / \mathrm{m} 22.2$ to 4.6 times less likely), renal complications (2.5 times more likely in NL and UK) and HbA1c $>8.5 \%$ (1.5-2 times more likely in ES and UK).

\section{2nd line}

Figure 3 shows the factors associated with 2 nd line therapies that reached the thresholds of analysis in all countries: SU, TZD or DPP4i in combination with metformin, each compared to any alternative 2nd line treatment. With advancing calendar years, the chance of receiving metformin combined with DPP4i increased (NL, IT, UK), whilst the chance of receiving the combination with TZD decreased strongly (NL, UK, ES) and with SU decreased a little (all). Advancing age decreased the chance of receiving DPP4i and TZD and increased the chance of receiving SU in combination with metformin. Combined metformin and TZD was negatively associated with cardiac drugs (NL, UK) coronary artery disease (UK, IT) and high CV risk (ES). Combined metformin and DPP4i was positively associated with high CV risk (ES) and negatively with renal complications (NL, UK). Higher BMI was associated with metformin combined with TZD (all) and DPP4i (NL, UK). High SBP decreased the chance of receiving combined metformin and DPP4i (NL, IT, UK). High HbA1c decreased the chance of receiving metformin with DPP4i (all) or TZD (NL). Compared to no prior treatment, associations of prior metformin use with metformin combination therapy were both negative (TZD: ES, NL; DPP4i: ES) and positive (TZD: IT; DPP4i: IT, NL).

\section{$\underline{3 r d ~ l i n e}$}

Figure 4 shows the factors associated with 3rd line therapies that reached the thresholds of analysis in all countries: triple therapy of metformin and SU combined with DPP4i, TZD or insulin and insulin combined with either metformin or SU. Together these covered a varied majority of all third line treatments per country (figure 1: NL 97\%, IT 57\%, ES 67\% and UK 78\%). 
Advancing age steadily increased the chance of receiving combined SU and insulin. Age $\geq 75$ years decreased the chances of metformin and SU combined with DPP4i (NL, IT) or TZD (NL, IT, ES). With the exception of combined metformin, SU and DPP4i therapy, advancing calendar years decreased the chance of receiving any of the SU containing treatments in all countries. Remarkably, in the UK the chance of receiving a combination of metformin with insulin also decreased over time. Renal complications and moderate to severe CKD increased the chance of receiving SU with insulin (NL, UK, ES), and decreased the chance of receiving metformin, SU and DPP4i (ES, IT) or TZD (all) triple therapy. Coronary artery disease and cardiac drugs reduced the chance of metformin-SU-TZD triple therapy (all) and metformin-SU-DPP4i triple therapy (NL, IT, ES) and slightly increased the chance of receiving insulin-oral therapy (IT, UK). High CV risk scores increased the chance of metforminSUDPP4i triple therapy in Spain. A BMI $\geq$ generally decreased the chance of insulin-oral combinations Higher BMI was mildly associated and any prior treatment (vs none) was more strongly associated with choice of metformin + SU + DPP4i. Older age slightly increased the chance of receiving metformin+SU+DPP4i in Spain and the UK, but decreased the chance in the Netherlands and Italy. Cardiac drugs (NL, IT, ES), cancer (NL, IT, ES), moderate to severe CKD (NL, IT, ES), renal complications (UK, IT) and $\mathrm{HbA} 1 \mathrm{C}>8.5 \%$ were negatively associated with the choice for metformin + SU + DPP4i. Age over 75 (NL, IT, ES), use of cardiac drugs (NL, IT, ES, UK) moderate to severe CKD (NL, $I T, E S)$, coronary artery disease (UK), cancer (UK, IT) and higher HbA1c (NL) reduced the likelihood of starting 3rd line treatment with metformin + SU + TZD. BMI $>25 \mathrm{~kg} / \mathrm{m} 2$, increased the likelihood, except for obese patients in the UK. Patients with prior treatments were generally more likely to receive this treatment than those without.

Patients with coronary artery disease (UK, IT), prior cancer (IT, ES, UK), higher HbA1c (all countries) and prior metformin treatment (ES, UK) were associated with receiving metformin + insulin. Higher $\mathrm{BMI}$ (NL, ES, UK) and any other prior treatment reduced the chance of receiving metformin + insulin. Prior use of metformin $+\mathrm{SU}$ was the factor most strongly associated with receiving metformin + SU + insulin. $\mathrm{HbA} 1 \mathrm{C}>8.5 \%$ and prior cancer were less strongly associated in all countries.

Increasing age, $\mathrm{HbA} 1 \mathrm{c}>8.5 \%, \mathrm{CKD}$ or renal complications, use of cardiac drugs and cancer were positively associated with $\mathrm{SU}+$ insulin as 3rd line treatment choice. High BMI and especially prior metformin + SU treatment were negatively associated in all countries.

$\underline{4 \text { th line }}$

The 100 patient threshold for analysis of other treatments than insulin was only met in the UK $(n=180)$ and Spain ( $n=248)$ for GLP-1ra monotherapy (figure 5$)$. The association of high BMI with GLP1ra use was so strong ( $0-3 \% \mathrm{BMI}<25 \mathrm{~kg} / \mathrm{m} 2,77-92 \% \geq 30 \mathrm{~kg} / \mathrm{m} 2$ in GLP-1ra users) that it had to be removed from the model to avoid convergence problems. Increased CV risk (ES) and advancing calendar years (UK) also increased the likelihood of receiving GLP-1ra monotherapy. Male gender (ES, UK), higher age (ES, UK), use of cardiac drugs (ES) coronary artery disease (UK), higher HbA1c (UK) and moderate to severe CKD or renal complications (ES, UK) reduced it.

\section{DISCUSSION}

Our study included all T2DM patients undergoing treatment intensifications in four European countries, which affected $79 \%$ of all patients treated with antidiabetic drugs in the 5 year study period. This suggests diabetes screening, monitoring and treatment modification is an ongoing process affecting the majority of patients. The fact that $47 \%$ of the study population consisted of patients who were initiated on first line treatment is testimony to the growing epidemic of T2DM. 
However, of all patients without immediate prior antidiabetic treatment $84 \%$ received 1st line treatment, $9 \%$ 2nd line, 3\% 3rd line and 5\% 4th line treatment. From a prior publication focusing on newly initiated patients 17 reporting similar proportions, it can be deduced that the majority of these are actually naïve to treatment rather than interrupting treatment or having treatment temporarily prescribed by a specialist, e.g. due to referral or hospitalisation. This is testimony that deviation from the rule of thumb of gradual treatment intensification is quite common, possibly because of high glucose levels due to longer duration of diabetes before start of treatment, or because diabetes was diagnosed and treatment started during hospitalisation, e.g. for a myocardial infarction.

Nevertheless, $84 \%$ of patients receive oral monotherapy as initial treatment, of which metformin is the first choice (79\%-94\%), emphasizing that guidelines 2,3 are being followed to a large extent.

More cardiovascular co-medication and co-morbidity were observed at intensifications to higher lines of treatment. This is in line with the expectation that patients with higher lines of treatment have a longer disease history, are older and thus have had a longer time to develop diabetic complications. However, there was no increase in the rate of co-morbidity and co-medication in those with a forth line therapy compared to third line despite a considerably higher mean age in this group.

Any treatment intensification is likely the result of inadequate glycemic control. Although in all databases $\mathrm{HbA1c}$ levels were frequently not recorded, the available information showed that a greater deviation from target resulted in specific treatment choices. For example, higher levels of $\mathrm{HbA1C}$ resulted in third line treatment choices that include insulin rather than triple oral therapy. This is in line with the 2006 ADA/EASD treatment algorithm and subsequent position statements2-4. For second line treatment high $\mathrm{HbA1c}$ levels significantly reduced the chance of receiving DPP4i or TZD in combination with metformin. At initiation of first line therapy high HbA1c levels increased the chance of receiving SU in all countries except the Netherlands. This result is in line with other observations in literature18.

Many of the associations we observed can be explained by the information on product labels. Patients receiving metformin in any treatment combination were generally more likely to have a high BMI, but are less likely to have higher age, use cardiac drugs (possibly associated with heart failure) or have renal complications. This is in line with the product label, which advises caution with renal failure, congestive heart failure requiring pharmacologic treatment and age over 80 years. Metformin and TZD both increase insulin sensitivity of cells and reduce hepatic glucose production, which helps prevent weight gain. TZD containing therapies were indeed also prescribed more frequently to patients with high BMI, but without cardiac drug use or coronary artery disease. TZD is contraindicated in patients with heart failure, especially in combination with insulin. On the other hand, patients receiving SU in any treatment combination were more likely to have renal complications, use cardiac drugs of have older age, and were less likely to have a high BMI. This indicates SU is used as an alternative for patients for whom metformin (and TZD) is not advised, especially if patients had renal failure and if the weight gain associated with SU use is deemed less problematic. SU stimulates insulin secretion from the pancreas, which results in weight gain and a high risk of hypoglycemia. Treatments including DPP4i were less often prescribed to patients with renal failure, for which dose adjustments are indicated, and more often to patients with high BMI, as it induces weight loss. Insulin was less often prescribed to patients with a high BMI, because it causes weight gain, but more often to patients with renal complications, for whom it is safe to use.

In view of the treatment algorithms, it makes sense that T2DM therapy immediately before intensification of treatment is associated with the choice of treatment. We found that those with metformin monotherapy or metformin-SU combination therapy often continued with an add-on, in 
line with the guidelines.

Drivers of treatment choice did differ per country, despite the general similarities described above. The actual choice is further motivated by the reimbursement practices and guideline recommendations in each country. The Dutch guidelines during the study period leave the least room for alternatives for metformin, SU and insulin5,19, which is reflected in all lines of treatment. The Italian and Spanish guidelines mention repaglinide as an alternative to SU7,8, which is reflected in the larger proportion of 'other' treatments in those countries. Reimbursement of GLP-1ra in the Netherlands was restricted to patients who failed other therapy, have a BMI $\geq 35 \mathrm{~kg} / \mathrm{m} 2$, prescription by a specialist and (since May 2011) no concomitant insulin20. Less stringent restrictions in combination with the larger sample sizes of the study populations in Spain and the UK meant that in these countries alternatives to fourth line insulin monotherapy reached the threshold for analysis, whereas they did not in the Netherlands and Italy. Strengths of associations also differed per country (see Figures 2-5), but this may be a reflection of the number of patients treated with alternative treatments as well as their characteristics, and the fact that different characteristics are included in the models of different countries, based on the backward selection of covariates.

Prescription preferences clearly changed over the study period. Concern around the safety of TZD use, specifically the withdrawal of rosiglitazone during the study period due to supposed cardiac risk, has resulted in a decline of TZD use over the years21,22. DPP4i gained market share at the expense of both TZD and SU in both 2nd and 3rd line treatments. Both the decline of SU and TZD and the rise of DPP4i are reflected in associations with calendar year in 2nd line treatment. Treatment of T2DM has continued to change since the study period with the introduction of new additions to the classes of DPP4i and GLP1ra and the introduction of several SGLT2 inhibitors.

There is little literature regarding factors associated with treatment choices. Maguire et al.18 studied the association between patient characteristics and the risk of treatment change after initiation of diabetes therapy, stratified by type of treatment change including augmentation (comparable to intensification to 2 nd and 3rd line triple oral therapy) and initiation of insulin (comparable to intensification to 3rd line oral-insulin combination therapy or 4th line insulin monotherapy). Contrary to our observation in third line treatment, they found that higher baseline $\mathrm{HbA1c}$ increased the chance of augmentation. Patients with metformin monotherapy were less likely to augment than those with SU monotherapy, contrary to our observation in second line.

\section{Strengths and limitations}

To our knowledge this is the first published study to compare treatment patterns and factors associated with choice of treatment intensifications across multiple countries. The use of electronic medical records allowed the evaluation of large numbers of patients. The common data model and standardised analysis tools promoted homogeneous analyses across all countries, making sure differences in patterns are not due to methodological differences.

The choice to combine treatments that were less frequently used across all countries into one group of 'other' treatments may have resulted in differences between the countries in the frequency and composition of this group. For example in Italy and Spain the use of repaglinide is more common than in the Netherlands or the UK. This may have influenced the effect of prior treatment in the analyses.

A limitation is the study period itself. The most recent 5 year period in each country differed slightly, some countries including 2012, others data up to 2011. The introduction of several members of new treatment classes such as GLP-1ra during the study period, combined with caution advocated by the 
guidelines, as well as the limited reimbursement for GLP-1ra in the Netherlands, meant limited numbers of patients and thus exclusion from analysis of factors associated with choice of GLP-1ra. The new class of Sodium-glucose co-transporter 2 (SGLT2) inhibitors were introduced after our study period and thus were not available for analyses.

Definition of lines of treatment is debatable. It can be argued that insulin only (4th line) may be less intense than insulin in combination with an OAD (3rd line). Also there was some switching back and forth between third and fourth line treatment, despite the very lenient definition of continuation of treatment classes when a repeat prescription was observed within 9 months. However, the fact that the users of fourth line treatment were older does seem to support the order of treatment lines.

A limitation is that primary care electronic patient records are not collected for research purposes. All comorbidities were based on diagnoses, examinations and hospitalisations as recorded by GPs, determined in the entire available history prior to the index date. Definitions were equal, but GP coding systems and ways of recording were different across countries, making comparison slightly less straightforward. Also, records may not be complete, e.g. some test results and specialist prescribed medications may be missing, and it is unclear whether missingness is random or possibly caused by unknown characteristics such as unwillingness to comply with treatment or disease management activities. Patients without known HbA1c values were significantly more often male, younger, and less often used lipid lowering and antihypertensive drugs. This implies that because of their relatively good health they were less closely monitored and therefore their $\mathrm{HbA1c}$ was not measured. Many unknown factors such as hypoglycemic events may also have influenced the frequency of $\mathrm{HbA1c}$ measurements and thus missingness of $\mathrm{HbA} 1 \mathrm{c}$ data. Because of the uncertainty we chose not to impute the missing data to avoid increasing possible bias. In stead we used missing values of clinical characteristics as a separate categorical value in the analyses. This likely has led to underestimation of the true associations between clinical characteristics such as BMI and $\mathrm{HbA1C}$ and the choice of treatment. Despite those possible sources of bias, the observed associations were in line with expectations, making this a minor limitation of our study.

\section{Conclusion}

The results suggest that the main factors driving treatment choice at any stage of intensification across four European countries are age, $\mathrm{HbA1c}$ level, renal comorbidity, macrovascular complications and BMI. These drivers are consistent with guidelines and contra-indications for specific medications. Differences between countries can be explained at least in part by differences in freedom of choice of treatment, based on guidelines and reimbursement policies.

\section{Acknowledgments}

The authors thank all the health care providers contributing information to the different databases included in this study. All authors contributed substantially to conception and design, acquisition of data, or analysis and interpretation of data. Dr. EB, Dr. AR, Drs. FH and Drs. GL contributed to the analysis of data. Dr. EH, Drs. JO, and Dr. IB drafted the manuscript and Dr. DPA, GH, FL and NH revised it critically for important intellectual content. All authors gave their final approval of the version of the final manuscript.

\section{Funding}

This work was supported by a grant from AstraZeneca Pharmaceuticals LP. No limitations were set with regard to the conduct of the studyand the writing of the manuscript by the study sponsors. 


\section{Conflicts of Interest statement}

Drs. JO, Dr. EH, Dr. IB and Dr. RH are employees of the PHARMO Institute for Drug Outcomes Research. This independent research institute performs financially supported studies for government and related healthcare authorities and several pharmaceutical companies. Dr. DPA's research group and/or department have received unrestricted research grants from Bioberica, AMGEN, Servier Laboratoires, and UCB Biopharma. Dr. GH has been a member of the Advisory Group of the THIN database and has received funding for research and consultancy from a number of pharmaceutical companies and from charities. Dr. FL provided consultancies in protocol preparation and data analysis for epidemiologic studies for AMGEN, Sanofi, and Eli-Lilly. Dr. NH is an employee of AstraZeneca. The authors have indicated that they have no other conflicts of interest regarding the content of this article. 


\section{REFERENCES}

1. International Diabetes Federation. IDF Diabetes Atlas, 7th edn. Brussels, Belgium: International Diabetes Federation, 2015.

2. Nathan DM, Buse JB, Davidson MB, et al. Medical management of hyperglycaemia in type 2 diabetes mellitus: a consensus algorithm for the initiation and adjustment of therapy: a consensus statement from the American Diabetes Association and the European Association for the Study of Diabetes. Diabetologia 2009; 52(1): 17-30.

3. Bailey T. Options for combination therapy in type 2 diabetes: comparison of the ADA/EASD position statement and AACE/ACE algorithm. The American journal of medicine 2013; 126(9 Suppl 1): S10-20.

4. Inzucchi SE, Bergenstal RM, Buse JB, et al. Management of hyperglycemia in type 2 diabetes, 2015: a patient-centered approach: update to a position statement of the American Diabetes Association and the European Association for the Study of Diabetes. Diabetes Care 2015; 38(1): 140-9.

5. Rutten GEHM, De Grauw WJC, Nijpels G, et al. NHG-Standaard Diabetes mellitus type 2 (Tweede herziening). Huisarts Wet 2006; 49(3): 137-52.

6. National Collaborating Centre for Chronic Conditions and the Centre for Clinical Practice at NICE. NICE guideline Type 2 diabetes: The management of type 2 diabetes, 2009.

7. Ariz Arnedo MJ, Artola Menéndez S, Barutell Rubio L, et al. Práctica clínica en la DM2. Barcelona, 2011.

8. (SID) AMDA-SIdD. Standard italiani per la cura del diabete mellito tipo 2, 2011. 9. AlvarezGuisasola F. Glycaemic control and implementation of the ADA/EASD-2006 consensus algorithm in type 2 diabetes mellitus patients in primary care in Spain. International journal of clinical practice 2014; 68(1): 28-39.

10. Baviera M, Monesi L, Marzona I, et al. Trends in drug prescriptions to diabetic patients from 2000 to 2008 in Italy's Lombardy Region: a large population-based study. Diabetes research and clinical practice 2011; 93(1): 123-30.

11. Overbeek JA, Heintjes EM, Prieto-Alhambra D, et al. Type 2 Diabetes Mellitus Treatment Patterns Across Europe: A Population-Based Multi-Database Study. Clinical therapeutics 2017.

12. PHARMO Institute for Drug Outcomes Research. 2016. http://www.pharmo.com (accessed 22 April 2016.

13. Garcia-Gil Mdel M, Hermosilla E, Prieto-Alhambra D, et al. Construction and validation of a scoring system for the selection of high-quality data in a Spanish population primary care database (SIDIAP). Inform Prim Care 2011; 19(3): 135-45.

14. Vinagre I, Mata-Cases $\mathrm{M}$, Hermosilla $\mathrm{E}$, et al. Control of glycemia and cardiovascular risk factors in patients with type 2 diabetes in primary care in Catalonia (Spain). Diabetes Care 2012; 35(4): 7749.

15. The Health Improvement Network (THIN) database. 2015. https://www.ucl.ac.uk/pcph/researchgroups-themes/thin-pub/database (accessed 29 April 2016. 
16. Zou G. A modified poisson regression approach to prospective studies with binary data. American journal of epidemiology 2004; 159(7): 702-6.

17. Overbeek JA, Heintjes EM, Hall GC, Lapi F, Prieto Alhambra D, Bezemer ID. Factors Associated with T2DM Treatment Choice Across Europe. Value Health 2015; 18(7): A600.

18. Maguire A, Mitchell BD, Ruzafa JC. Antihyperglycaemic treatment patterns, observed glycaemic control and determinants of treatment change among patients with type 2 diabetes in the United Kingdom primary care: a retrospective cohort study. BMC endocrine disorders 2014; 14: 73.

19. Verduijn T, Janssen PG. NHG-Standpunt DPP-4-remmers en GLP-1-agonisten. 2010. http://www.diabetes2.nl/uploads/user/rest/DPP-4-remmers_en_GLP-1-agonisten.pdf (accessed April 22, 2016.

20. Medicijnbalans, GLP-1-agonisten. Vergoeding. In: Instituut voor Verantwoord Medicijngebruik, editor.; 2017.

21. Leal I, Romio SA, Schuemie M, Oteri A, Sturkenboom M, Trifiro G. Prescribing pattern of glucose lowering drugs in the United Kingdom in the last decade: a focus on the effects of safety warnings about rosiglitazone. Br J Clin Pharmacol 2013; 75(3): 861-8.

22. Tzoulaki I, Molokhia M, Curcin V, et al. Risk of cardiovascular disease and all cause mortality among patients with type 2 diabetes prescribed oral antidiabetes drugs: retrospective cohort study using UK general practice research database. BMJ (Clinical research ed) 2009; 339: b4731. 


\section{TABLES \& FIGURES}

Table 1 General characteristics at intensification date of patients treated for T2DM per country Figure 1 Proportion of patients on specific T2DM treatments per treatment line per country Figure 2 Factors across countries associated with choice of SU as first line therapy Figure 3 Factors across countries associated with choice of common second line therapies Figure 4 Factors across countries associated with choice of common third line therapies Figure 5 Factors across countries associated with choice of common fourth line therapies 
Table 1 General characteristics at intensification date of patients treated for T2DM per country

\begin{tabular}{|c|c|c|c|c|}
\hline & $\begin{array}{c}\text { First line } \\
N=218,176 \\
n(\%)\end{array}$ & $\begin{array}{c}\text { Second line } \\
\mathrm{N}=138,613 \\
\mathrm{n}(\%)\end{array}$ & $\begin{array}{c}\text { Third line } \\
N=96,412 \\
n(\%)\end{array}$ & $\begin{array}{c}\text { Fourth line } \\
\mathrm{N}=32,369 \\
\mathrm{n}(\%)\end{array}$ \\
\hline \multicolumn{5}{|l|}{ Country } \\
\hline Netherlands & $17,393(8)$ & $9,966(7)$ & $5,971(6)$ & $2,287(7)$ \\
\hline Italy & $20,554(9)$ & $11,005(8)$ & $8,775(9)$ & $4,832(15)$ \\
\hline Spain & $126,745(58)$ & $86,081(62)$ & $60,890(63)$ & $21,485(66)$ \\
\hline UK & $53,484(25)$ & $31,111(22)$ & $20,776(22)$ & $3,765(12)$ \\
\hline \multicolumn{5}{|l|}{ Gender } \\
\hline Male & $112,341(51)$ & $71,401(52)$ & $49,066(51)$ & $16,623(51)$ \\
\hline Female & $105,835(49)$ & $66,762(48)$ & $47,346(49)$ & $15,746[49]$ \\
\hline \multicolumn{5}{|l|}{ Age (years) } \\
\hline$>18-50$ & $38,428(18)$ & $18,040(13)$ & $11,064(11)$ & $3,921(12)$ \\
\hline$>50-65$ & $82,726(38)$ & $52,891(38)$ & $34,695(36)$ & $8,366(26)$ \\
\hline$>65-75$ & $55,098(25)$ & $37,823(27)$ & $27,525(29)$ & $8,485(26)$ \\
\hline$>75$ & $41,924(19)$ & $29,409(21)$ & $23,128(24)$ & $11,597(36)$ \\
\hline \multicolumn{5}{|l|}{ Year of intensification date } \\
\hline 2007 & $33,504(15)$ & $18,089(13)$ & $9,411(10)$ & $5,939(18)$ \\
\hline 2008 & $44,223(20)$ & $24,436(18)$ & $14,122(15)$ & $7,031(22)$ \\
\hline 2009 & $44,385(20)$ & $25,468(18)$ & $16,805(17)$ & $6,919(21)$ \\
\hline 2010 & $45,467(21)$ & 26,424 (19) & $18,387(19)$ & $6,936(21)$ \\
\hline 2011 & $40,542(19)$ & $36,386(26)$ & $32,161(33)$ & $5,173(16)$ \\
\hline 2012 & $10,055(5)$ & $7,360(5)$ & $5,526(6)$ & $371(1)$ \\
\hline \multicolumn{5}{|l|}{ Prior antidiabetic treatment" } \\
\hline$\underline{\text { None }}$ & $218,176(100)$ & $23,004(17)$ & $7,479(8)$ & $12,064(37)$ \\
\hline \multicolumn{5}{|l|}{ Prior to second line } \\
\hline Metformin & - & $78,470(57)$ & - & - \\
\hline su & - & $31,170(23)$ & - & - \\
\hline TZD & - & $684(<0.5)$ & - & - \\
\hline DPP4i & - & $817(1)$ & - & - \\
\hline \multicolumn{5}{|l|}{ Prier to third line } \\
\hline Metformin + sU & - & - & $46,846(49)$ & - \\
\hline$T Z D+O A D$ & - & - & $6,502(7)$ & - \\
\hline$D P P 4 i+O A D$ & - & - & $9,198(10)$ & - \\
\hline OAD monotherapy & - & - & $20,321(21)$ & $6,488(20)$ \\
\hline $2 O A D$ & - & - & - & $2,961(9)$ \\
\hline $3 O A D$ & - & - & - & $325(1)$ \\
\hline \multicolumn{5}{|l|}{ Prior to fourth line } \\
\hline OAD + insulin & - & - & - & $10,324(32)$ \\
\hline$O A D+G L P-1 r a$ & - & - & - & $160(<0.5)$ \\
\hline $\begin{array}{l}\text { other* grouping of remaining treatments } \\
\text { (present }<5 \% \text { ) }\end{array}$ & - & $4,018(3)$ & $6,066(6)$ & $47(<0.5)$ \\
\hline \multicolumn{5}{|l|}{ Co-medication". } \\
\hline Lipid lowering drugs & $78,897(36)$ & $72,411(52)$ & $56,657(59)$ & $13,713(42)$ \\
\hline Antihypertensive drugs & $122,223(56)$ & $88,471(64)$ & $68,461(71)$ & $21,292(66)$ \\
\hline Cardiac drugs & $19,418(9)$ & $13,971(10)$ & $12,201(13)$ & $6,021(19)$ \\
\hline Anticoagulants & $55,880(26)$ & $49,117(36)$ & $43,729(45)$ & $14,368(44)$ \\
\hline COPD/asthma drugs & $27,973(13)$ & $16,829(12)$ & $12,988(13)$ & $5,476(17)$ \\
\hline
\end{tabular}

Microvascular comorbidities 


\begin{tabular}{|c|c|c|c|c|}
\hline & $\begin{array}{c}\text { First line } \\
\begin{array}{c}N=218,176 \\
n(\%)\end{array}\end{array}$ & $\begin{array}{l}\text { Second line } \\
\begin{array}{c}N=138,613 \\
n(\%)\end{array}\end{array}$ & $\begin{array}{c}\text { Third line } \\
\mathrm{N}=96,412 \\
\mathrm{n}(\%)\end{array}$ & $\begin{array}{c}\text { Fourth line } \\
\begin{array}{c}N=32,369 \\
n(\%)\end{array}\end{array}$ \\
\hline Diabetic foot/PVD/neuropathy & $10,332(5)$ & $10,092(7)$ & $9,447(10)$ & $2,997(9)$ \\
\hline Retinopathy & $3,838(2)$ & $6,678(5)$ & $8,424(9)$ & $2,566(8)$ \\
\hline Renal complications & $9,289(4)$ & $8,110(6)$ & $8,458(9)$ & $4,711(15)$ \\
\hline \multicolumn{5}{|l|}{ Macrovascular comorbidities } \\
\hline Coronary artery disease & $13,633(6)$ & $10,000(7)$ & $8,799(9)$ & $3,923(12)$ \\
\hline Cerebrovascular disease & $7,687(4)$ & $5,307(4)$ & $4,888(5)$ & $2,645(8)$ \\
\hline \multicolumn{5}{|l|}{ Other comorbidities } \\
\hline Ulcers at other locations & $947(<0.5)$ & $736(1)$ & $813(1)$ & $545(2)$ \\
\hline Urinary tract infections & $17,697(8)$ & $11,874(9)$ & $10,174(11)$ & $3,471(11)$ \\
\hline Other cardiovascular disease & $76,966(35)$ & $50,062(36)$ & $36,443(38)$ & $11,957(37)$ \\
\hline Chronic respiratory disease & $19,126(9)$ & $11,808(9)$ & $9,429(10)$ & $3,494(11)$ \\
\hline Cancer & $12,716(6)$ & $8,662(6)$ & $7,295(8)$ & $3,530(11)$ \\
\hline Mental disease & $45,203(21)$ & $29,750(22)$ & $23,079(24)$ & $7,704(24)$ \\
\hline \multicolumn{5}{|l|}{ CKD } \\
\hline Normal to stage $\| * * *$ & $205,247(94)$ & $129,053(93)$ & $88,026(91)$ & $27,618(85)$ \\
\hline Stage III, IV, V & $12,929(6)$ & $9,110(7)$ & $8,386(9)$ & $4,751(15)$ \\
\hline \multicolumn{5}{|l|}{ Cardiovascular risk assessment } \\
\hline Low risk & $19,204(9)$ & $13,341(10)$ & $9,615(10)$ & $2,108(7)$ \\
\hline Moderate risk & $17,262(8)$ & $18,294(13)$ & $14,615(15)$ & $2,533(8)$ \\
\hline High risk & $8,996(4)$ & $10,095(7)$ & $8,330(9)$ & $1,635(5)$ \\
\hline Unknown & $165,939(76)$ & $92,278(67)$ & $61,008(63)$ & 25,716 (79) \\
\hline \multicolumn{5}{|l|}{ HbA1c (\%) ${ }^{* *}$} \\
\hline$<7.5$ & $55,679(26)$ & $25,685(19)$ & $12,324(13)$ & $4,456(14)$ \\
\hline $7.5-8.5$ & $24,792(11)$ & $26,193(19)$ & $17,127(18)$ & $2,563(8)$ \\
\hline$>8.5$ & $25,372(12)$ & $29,096(21)$ & $27,406(28)$ & $5,122(16)$ \\
\hline Unknown & $112,333(51)$ & $57,189(41)$ & $39,555(41)$ & $20,228(62)$ \\
\hline \multicolumn{5}{|l|}{ BMI $(\mathrm{kg} / \mathrm{m} 2)^{*}$} \\
\hline$<25$ & 19,409 (9) & $14,723(11)$ & $11,915(12)$ & $5,329(16)$ \\
\hline $25-<30$ & $64,959(30)$ & $46,241(33)$ & $31,469(33)$ & $9,227(29)$ \\
\hline$\geq 30$ & $98,731(45)$ & $60,969(44)$ & $43,017(45)$ & $10,658(33)$ \\
\hline Unknown & $35,077(16)$ & $16,230(12)$ & $10,011(10)$ & $7,155(22)$ \\
\hline \multicolumn{5}{|l|}{$\mathrm{SBP}(\mathrm{mmHg})$} \\
\hline$\leq 140$ & $89,496(41)$ & $63,304(46)$ & $43,561(45)$ & $11,524(36)$ \\
\hline$>140$ & $43,715(20)$ & $28,397(21)$ & $19,408(20)$ & $5,156(16)$ \\
\hline Unknown & 84,965 (39) & $46,462(34)$ & $33,443(35)$ & $15,689(48)$ \\
\hline \multicolumn{5}{|l|}{$\mathrm{DBP}(\mathrm{mmHg})$} \\
\hline$\leq 90$ & $118,857(54)$ & $84,296(61)$ & $58,767(61)$ & 15,706 (49) \\
\hline$>90$ & $14,307(7)$ & $7,381(5)$ & $4,188(4)$ & $974(3)$ \\
\hline Unknown & $85,012(39)$ & $46,486(34)$ & $33,457(35)$ & $15,689(48)$ \\
\hline
\end{tabular}

- treatment immediately preceding intensification; ${ }^{* *}$ determined in the 6 months prior to index date; ${ }^{* *}$ determined in the entire available history; 2007 without UK, 2012 UK only 


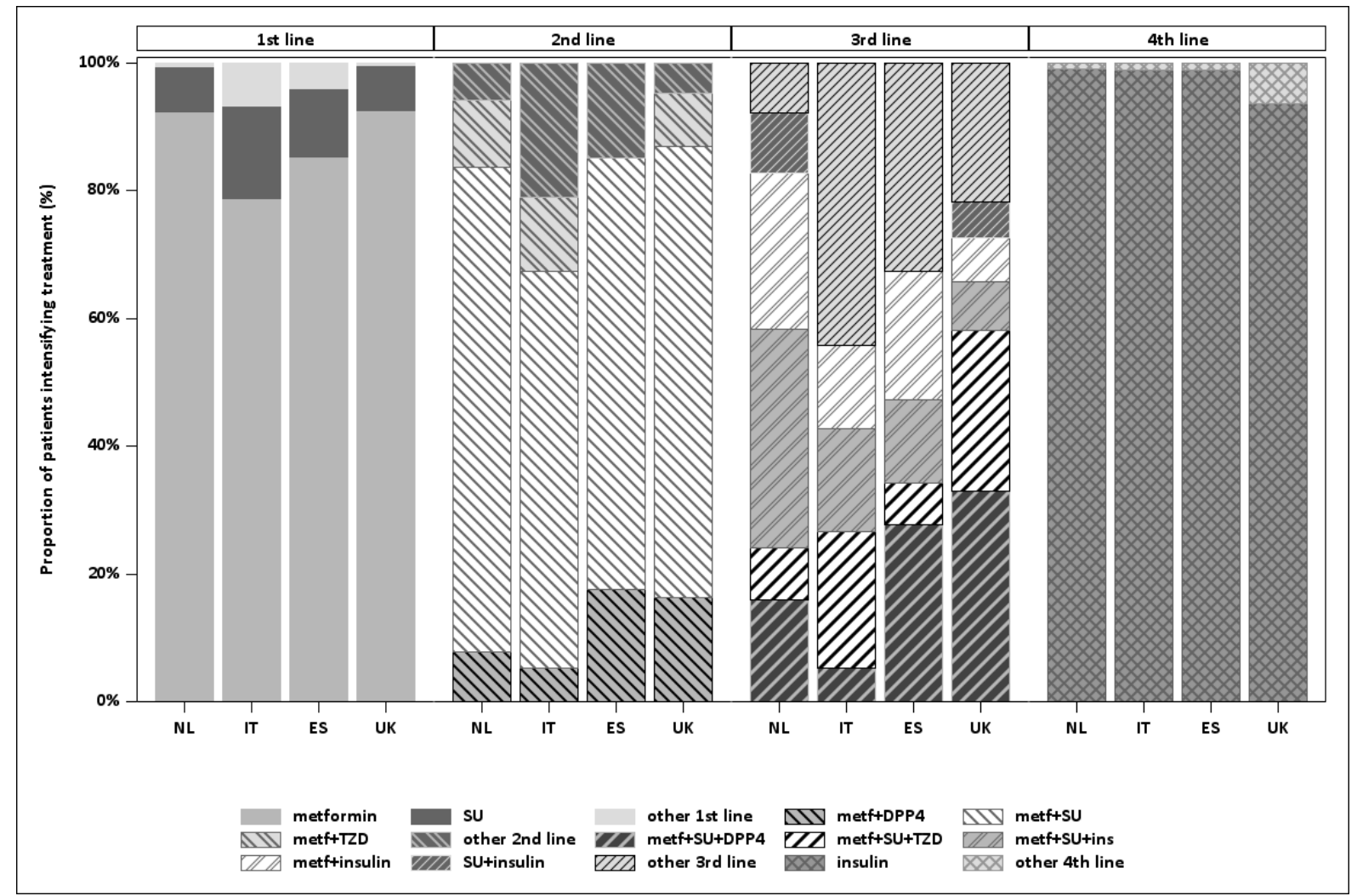

Figure 1 Proportion of patients on specific T2DM treatments per treatment line per country 


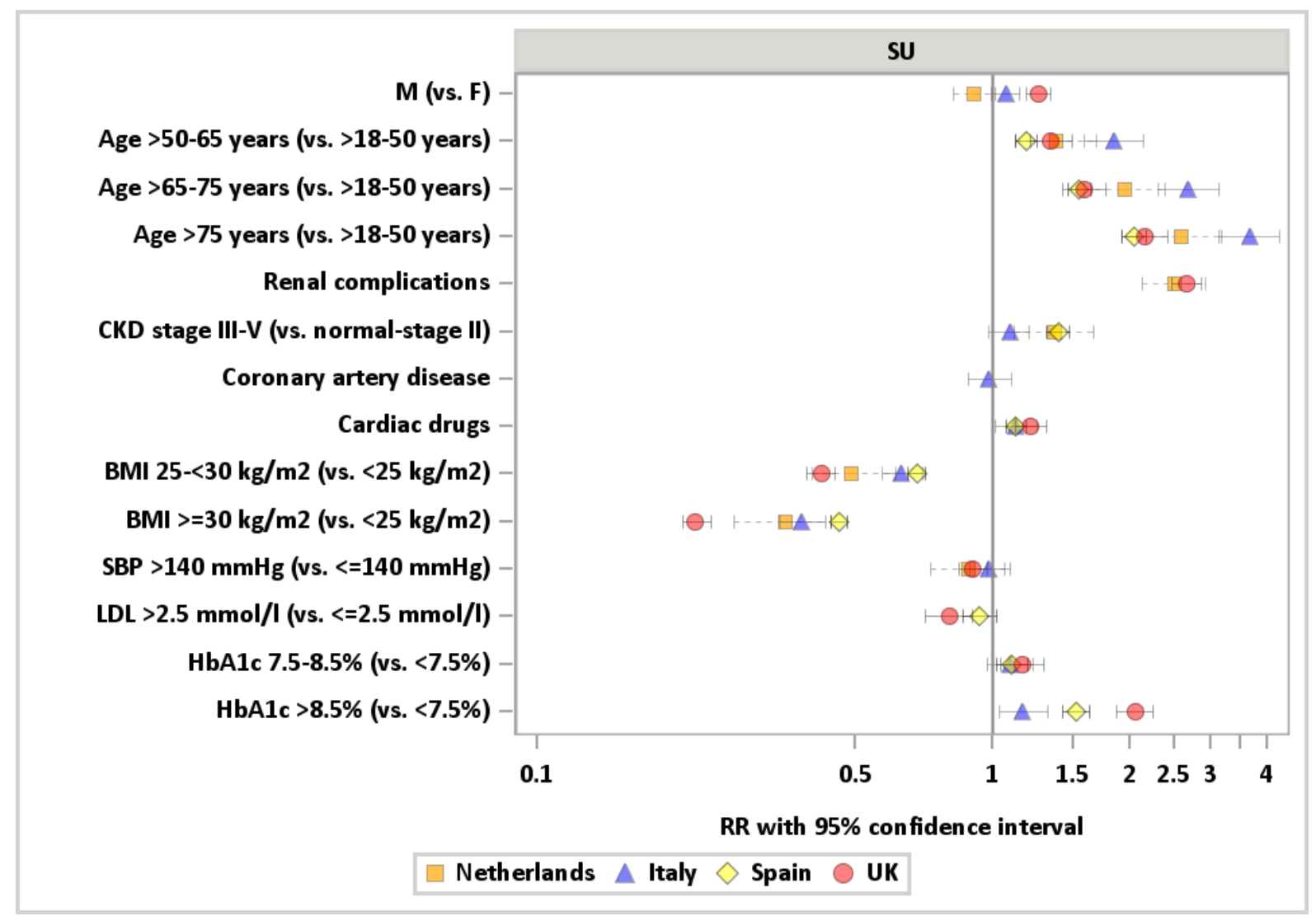

Figure 2 Factors across countries associated with choice of SU as first line therapy 


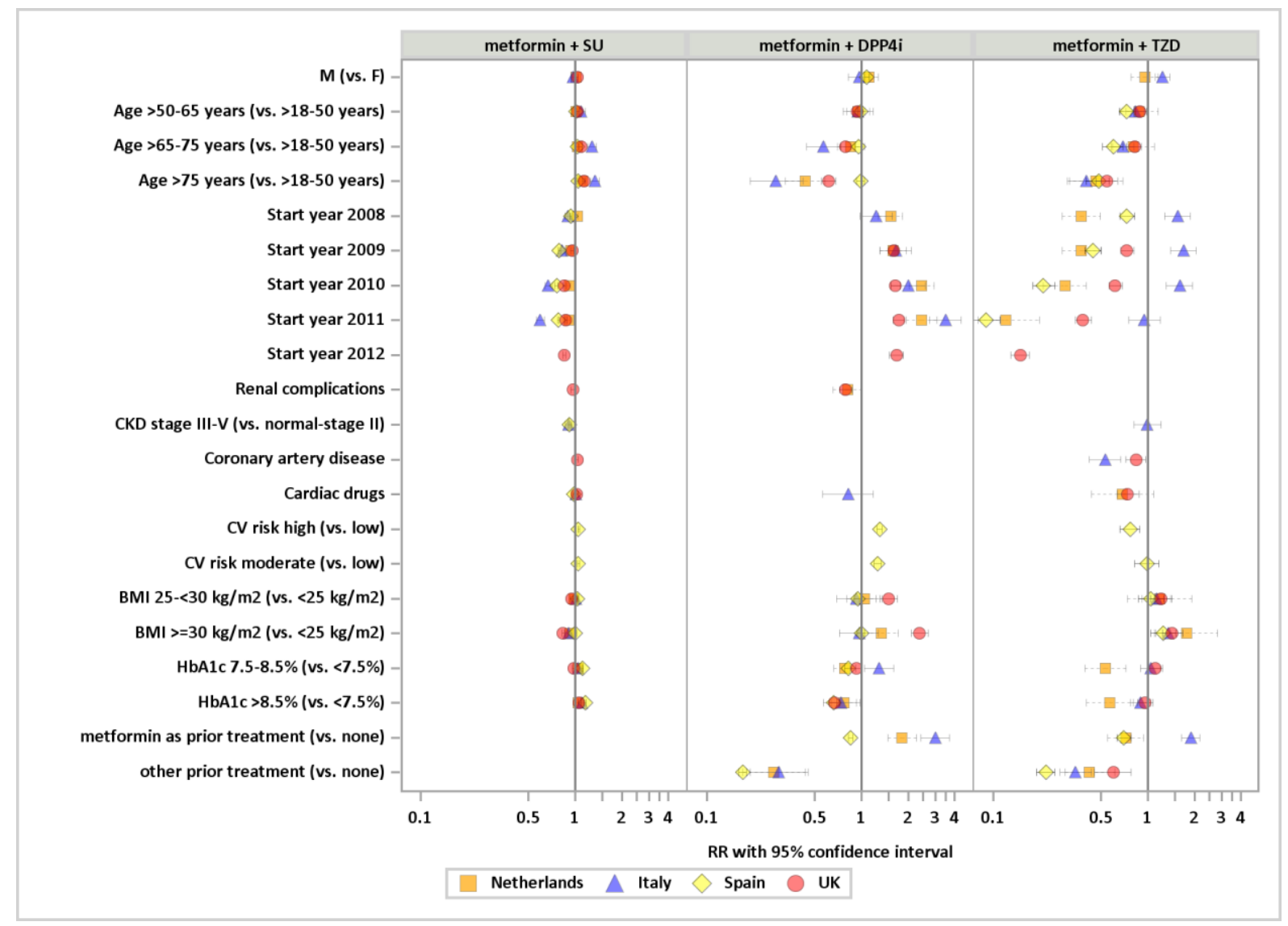

Figure 3 Factors across countries associated with choice of common second line therapies 


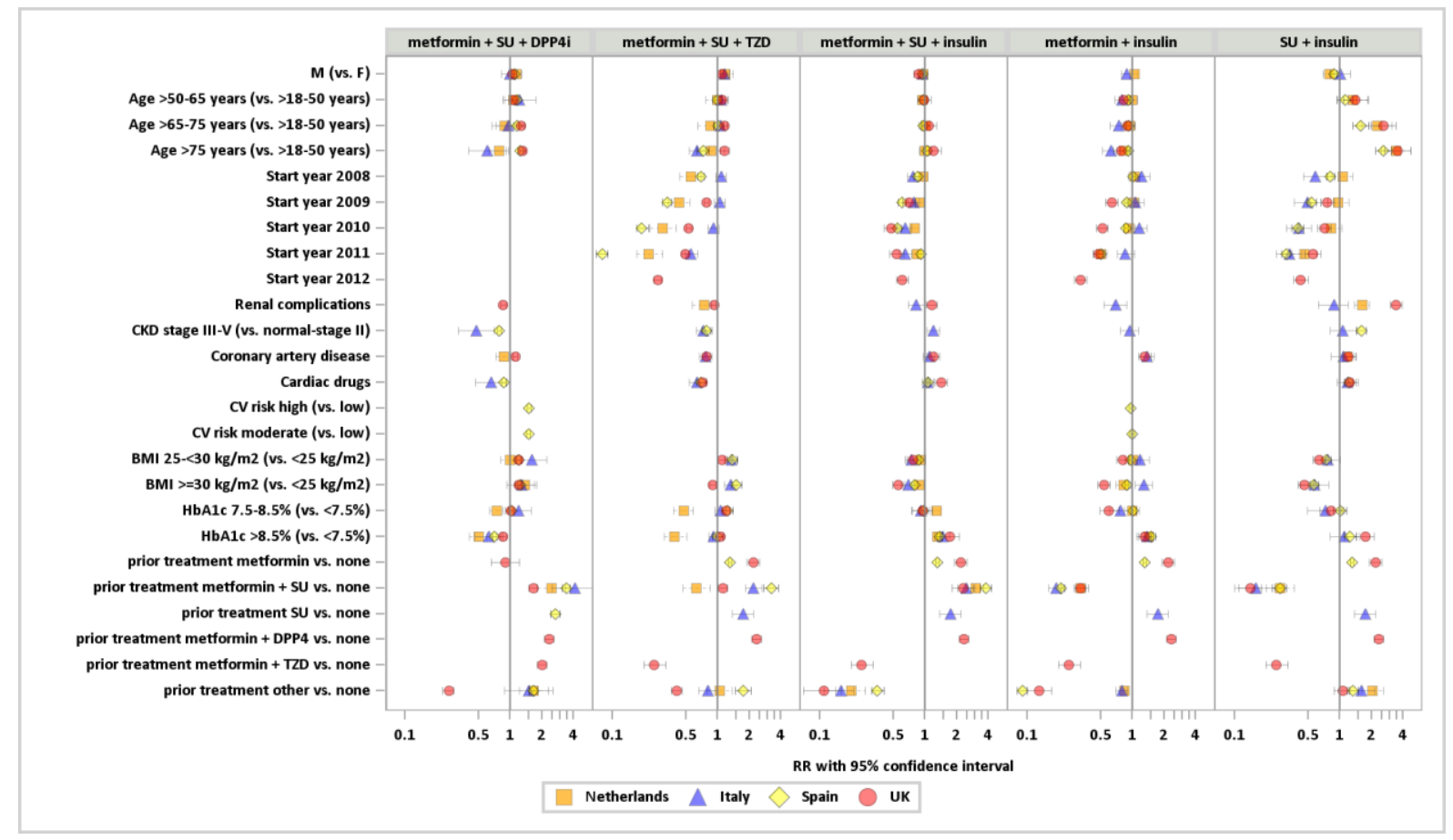

Figure 4 Factors across countries associated with choice of common third line therapies 


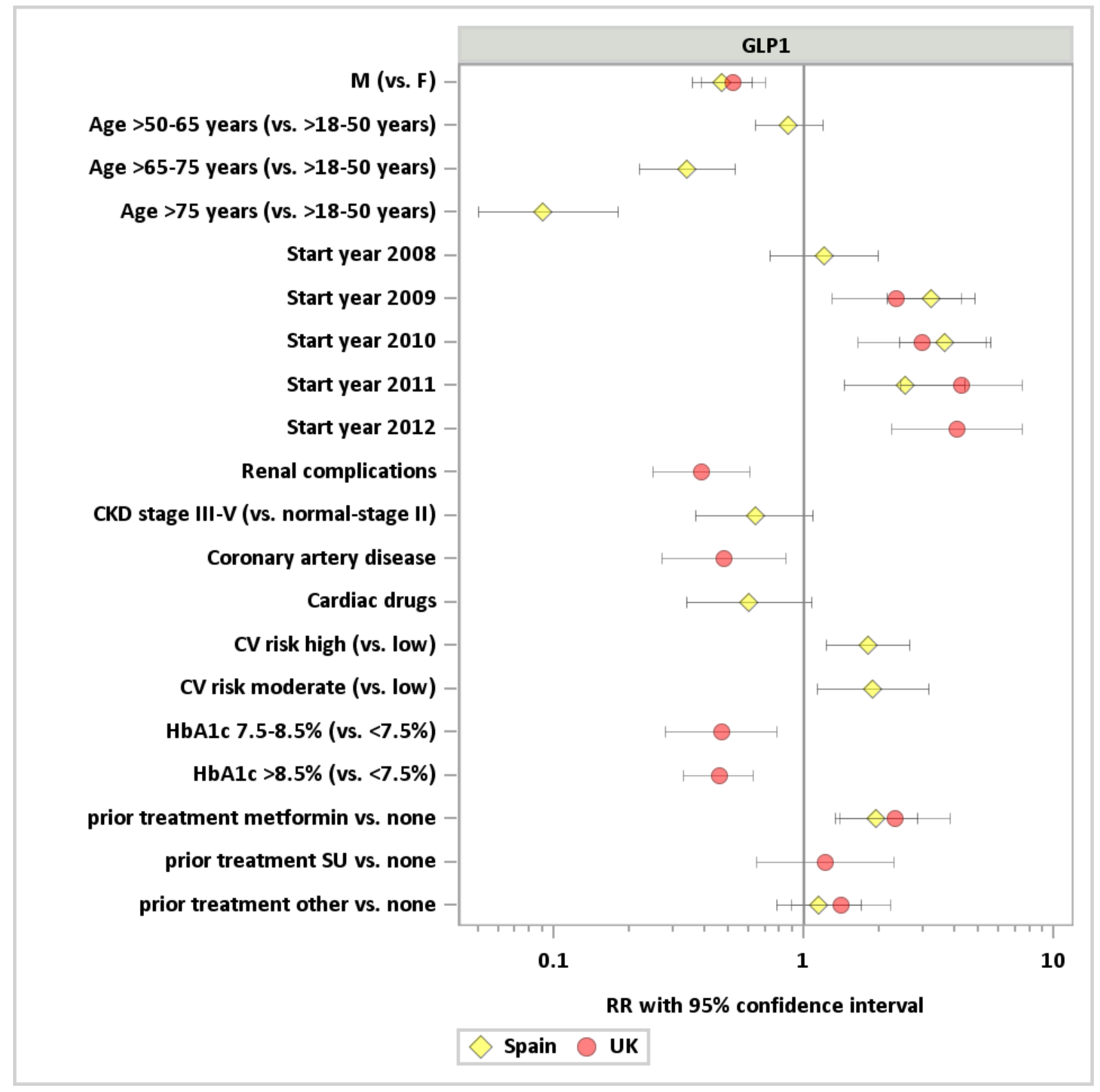

Figure 5 Factors across countries associated with choice of common fourth line therapies 